\title{
Group Orbits of GPS Satellite Configurations for Constellation Management
}

\author{
Dale C. Peterson ${ }^{1}$ and Mark F. Storz ${ }^{2}$ \\ 1. United States Air Force Academy, USAF Academy, Colorado, 80840 \\ 2. Headquarters, Air Force Space Command, Peterson AFB, Colorado, 80914
}

Received: June 05, 2016 / Accepted: July 03, 2016 / Published: January 25, 2017.

\begin{abstract}
Air Force Space Command is interested in improving the accuracy of GPS receiver positioning, navigation, and timing. To this end, it is useful to identify a set of optimal satellite constellations where each corresponds to a configuration specifying the number of satellites in each orbital plane. These constellations could then be maintained in a library for future use as satellites fail and are launched. We utilize symmetry in the geometry of the GPS satellite orbits to partition the configurations into a much smaller set of equivalence classes where each class has the same overall receiver accuracy performance. We apply a classical algebraic combinatorial result, Pólya's Theorem, to count and categorize the classes. Incorporating our results into a GPS constellation optimization computer tool will reduce run time by about an order of magnitude. We apply other algebraic and combinatorial techniques in original ways to count the class sizes and the classes that contain a given number of satellites. Finally, we break the equivalence classes into a still smaller set of new "structure" classes that are useful in applying the GPS computer tool.
\end{abstract}

Key words: Global Positioning System (GPS), satellite constellation design, dihedral group, Pólya’s Theorem

\section{Introduction}

In this paper we partition the Global Positioning System (GPS) satellite orbit configurations into a set of equivalence classes where the constellations in each class have the same overall receiver accuracy performance. Our approach yields a number of other results that will assist in satellite constellation design.

Terms in this paper that are italicized have been defined and used elsewhere, or the italics are for emphasis. Terms that are boldly italicized are new to the literature in the context of this paper's topics, to the best of our knowledge.

We begin by presenting the relevant GPS background.

\subsection{Overview of the GPS Constellation and Performance}

A GPS constellation, a set of satellites working together to support the positioning, navigation, and

Corresponding author: Dale Peterson, Professor of Mathematics, research field: operations research, discrete mathematics, space systems. E-mail: dale.peterson@usafa.edu. timing (PNT) mission, consists of between 24 and 31 satellites placed in six nearly equally-spaced orbital planes. The planes are labeled A, B, C, D, E, and F; each is inclined at about $55^{\circ}$ from the equatorial plane; and each satellite orbit is nearly circular. See Figure 1.

We use the term constellation to denote a specific number of satellites in each GPS orbital plane and their placement within each plane. We use configuration to denote an assignment of the number of satellites to each orbital plane, without regard to their placement. For example, the configuration 565544 means there are five satellites in plane A, six in plane B, and so on.

A GPS satellite has an orbital period of one-half of a sidereal day and a ground track cycle of a full sidereal day; see Figure 2. A sidereal day is the time it takes the earth to rotate $360^{\circ}$ with respect to the background stars and is about 23 hours and 56 minutes. In this paper, any reference to "day" means a sidereal day. The entire GPS geometry relative to the rotating earth has a period of one day. 


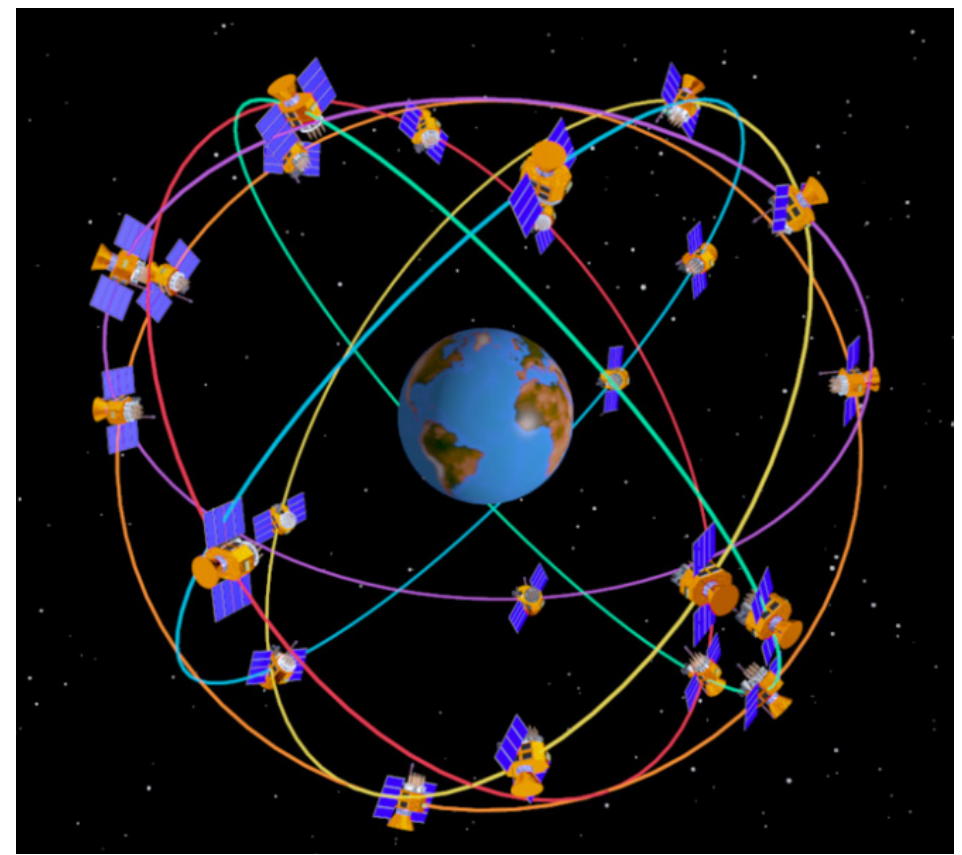

Fig. 1 The nominal 24-satellite GPS constellation (figure available at http://www.gpstk.org/bin/view/Documentation/LinuxJournalPaperSeptember2004).

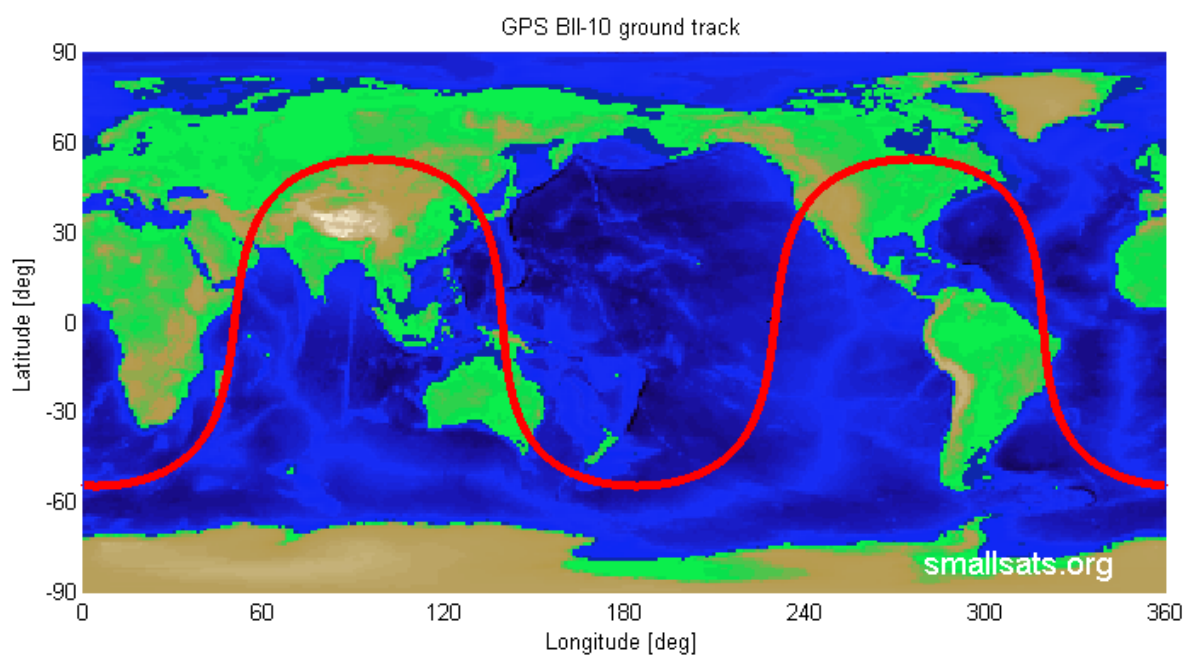

Fig. 2 Ground track of an example GPS satellite over a day (figure available at http://smallsats.org/tag/Earth-topographic-map/).

The accuracy of a GPS navigation solution at an Earth location at an instant in time depends on the geometry of the satellites visible from the location at that time. Metrics commonly used are Dilution of Precision (DOP) [1,2]. The smaller the DOP value, the more accurate the position or time determined by the GPS receiver. Overall performance of a GPS constellation is typically measured as some function of the DOP values over space (earth locations) and time (a GPS period of a day). See [3] for a discussion of several such functions.

Figure 3 is a coverage map for a particular constellation. It displays the outage for each geographic location: the time (in bins, e.g. 15-30 minutes) over a day each location's Position DOP (PDOP) exceeds some threshold - in this case, PDOP $>3$. (Most GPS 


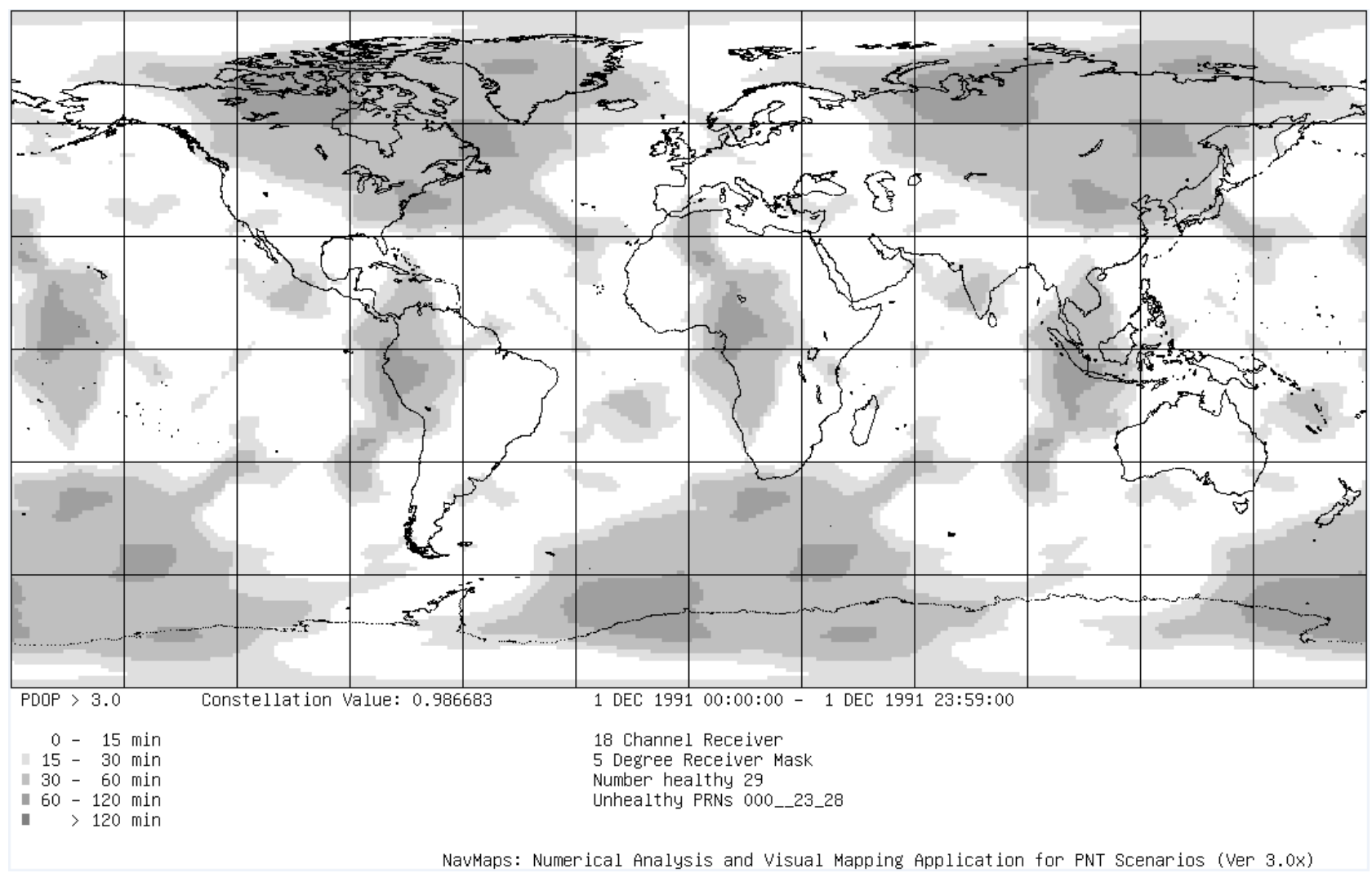

Fig. 3 Coverage map for a 565544 configuration.

PDOP values are between 1 and 4.) This map was generated by a computer tool called NAVMAPS (Numerical Analysis and Visual Mapping Application for PNT Scenarios), a discrete space-time grid simulation in which navigation solutions are computed at representative points on the earth at time steps over a day. See [3] for more on NAVMAPS.

Observe that the coverage pattern on the longitude interval $0^{\circ}-180^{\circ}$ is repeated on the interval $180^{\circ}-360^{\circ}$. This occurs because each satellite ground track has a wavelength of $180^{\circ}$ longitude over one-half of a day and all of the satellites are progressing along their respective ground tracks at the same rate. Thus, two GPS constellations that are the same except phase-shifted by three orbital planes $\left(180^{\circ}\right)$ produce the same coverage map.

Information on the precise outage for each geographic location is computed in the simulation and stored in the background of the coverage maps. This information is an important input into GPS performance prediction tools, which generate long-term measures of performance based on potential constellations predicted to occur in the future. Such tools are used to study alternative constellation management strategies. A typical performance prediction tool computes the expected value of outage at each earth location based on the contribution of all possible coverage maps at some given time in the future. Each coverage map's predicted probability is the sum of the predicted probabilities of the two $180^{\circ}$ phase-shifted constellations that produce that coverage map. This results in a single aggregate coverage map of the predicted navigational accuracy.

Performance prediction tools are run repeatedly as satellites fail or their life expectancies change, launches are scheduled, and other operational factors occur that affect constellation probabilities over time. Theoretically, there are an infinite number of constellations that could be input into a performance prediction tool. A reasonably-sized representative set 
must somehow be selected to render the problem tractable.

One of the motivations of our work is to identify a relatively small set of constellations that are operationally desirable and which will be input into a performance prediction tool. This can result in enormous savings in computer run and analysis time of future GPS performance, as well as considerably improved management of possible future constellations.

\subsection{Constellation Design Tool and Assumptions}

A computer tool called GPS Simulation and Optimization Tool (GPSOPT) was introduced in [3] that, given a configuration, searches for its unique optimal constellation. GPSOPT includes two components. The first is a space-time grid simulation like NAVMAPS. The second is a nonlinear program that inputs a user-selected objective performance function and one or more initial constellations from which to begin its search. The nonlinear program requires many performance function evaluations, each requiring a run of the simulation. So, optimizing for even one configuration is computationally intensive. A goal in our study is to reduce the number of configurations that need to be optimized.

GPSOPT makes the following assumptions:

- The earth is a perfect sphere. In particular, all Earth locations are at the same distance from the earth's center.

- The orbits are circular with constant velocity and a period of one-half of a (sidereal) day.

- The six orbital planes are equally spaced with a difference of $60^{\circ}$ between consecutive planes, and each is inclined at $55^{\circ}$.

- Navigation solutions and constellation performance are based entirely on satellite-earth geometry.

- The mask angle, the elevation angle above the horizon at which a satellite becomes visible to a location on the earth, is the same everywhere.
(GPSOPT uses $5^{\circ}$ as a default.)

- All Earth locations are treated the same in computing constellation performance. That is, we do not treat locations differently for geographic, political, strategic, or other operational reasons.

These assumptions are operationally reasonable for general studies [3]. We use them throughout the paper.

Once GPSOPT finds unique constellations for all configurations, they can be maintained in a library for use in performance prediction tools and for future operational use.

\section{Classes of Configurations}

The U. S. Air Force Space Command, which manages the GPS, is interested in examining configurations in which each of the six orbital planes contains either 4,5 , or 6 satellites. This yields $3^{6}$, or 729 possible configurations. But symmetry resulting from the assumptions of Subsection 1.2 reduces the number of configurations that need to be studied. For example, Figures 3, 4, and 5 show coverage maps for constellations having the configurations 565544, 655445 (565544 shifted, or "rotated" to the left, with wrap-around), and 445565 ("reflection" of 565544). We kept the same satellite placements, or arguments of latitude - angle along the orbit from the equatorial plane at a given instant in time - in corresponding phase-shifted (rotated) orbital planes; to preserve the same "mirror image" coverage pattern for the reflected configuration, the signs of the arguments of latitude must be reversed. Notice the coverage patterns that look like "turkeys on a serving plate" in Figure 3 are shifted $60^{\circ}$ to the west in Figure 4, and Figure 5 is a map of the "mirror image" as indicated by the "drumsticks" pointing to the west instead of the east.

In fact, Figures 3, 4, and 5 are the same except they are shifted or reflected. The three constellations yield the same overall performance. (This assumes that all Earth locations are treated equally; see the assumptions in Subsection 1.2.) Indeed, all configurations that can be obtained from 565544 by any combination of rotations 


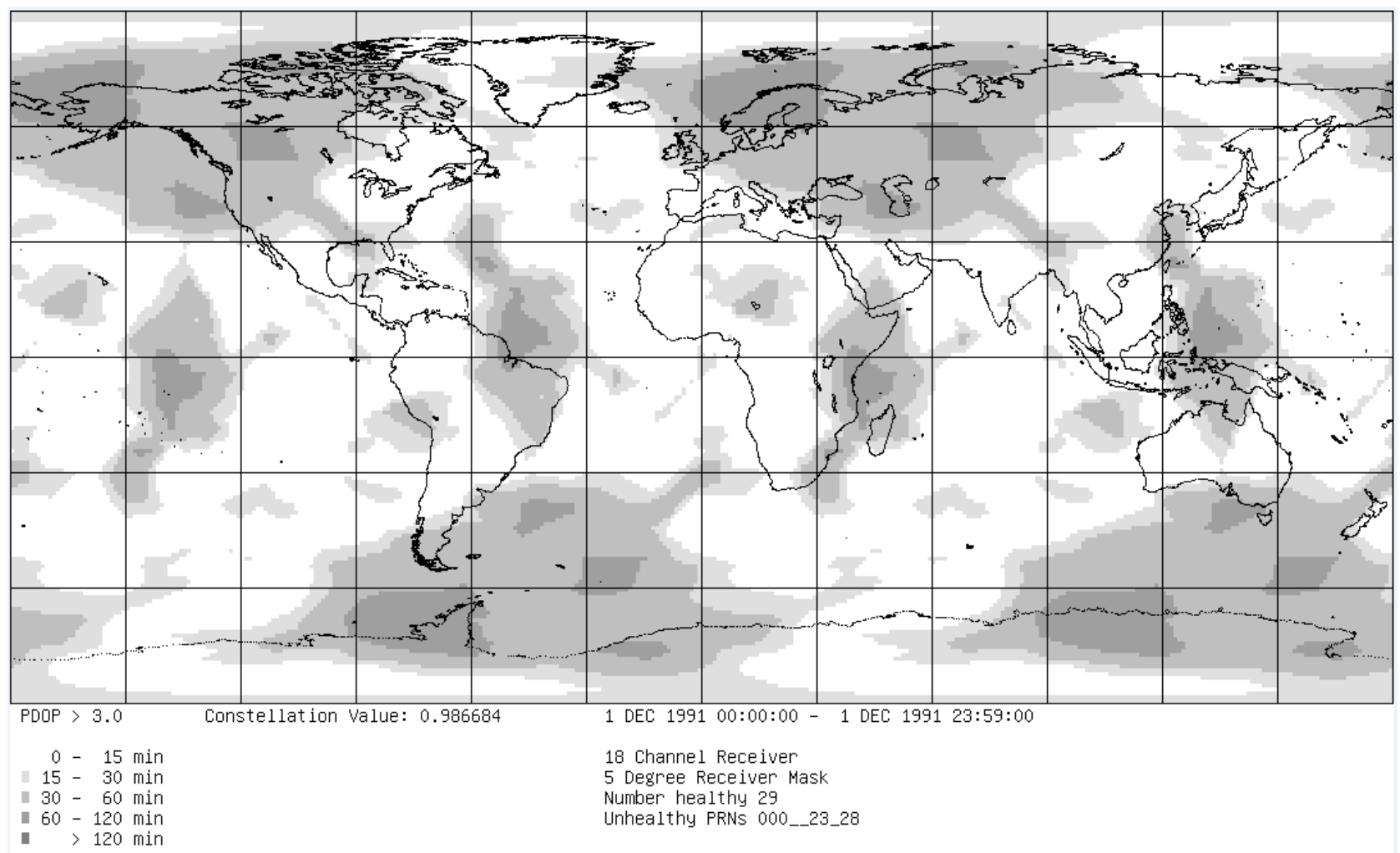

NavMaps: Numerical Analysis and Visual Mapping Application for PNT Scenarios (Ver 3.0x)

Fig. 4 Coverage map for a 655445 configuration (565544 shifted $60^{\circ}$ to the west).

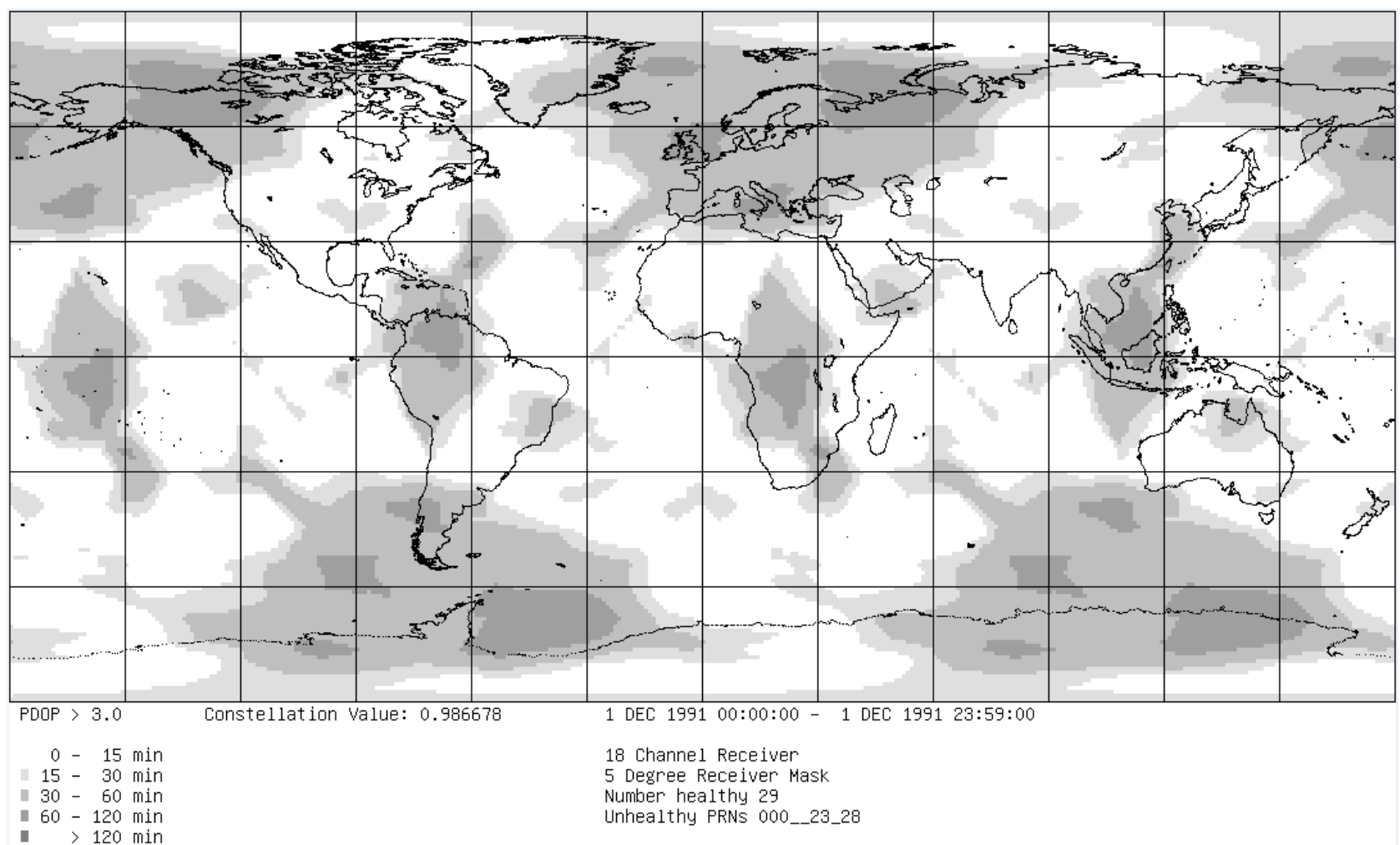

NavMaps: Numerical Analysis and Visual Mapping Application for PNT Scenarios (Ver 3.0x)

Fig. 5 Coverage map for a 445565 configuration (mirror image of 565544). 
(orbital plane phase-shifts) and reflections (plane reversals) are in the same equivalence class: constellations for those configurations have the same performance, when keeping the satellite arguments of latitude in corresponding orbital planes as described above.

In order to identify an optimal satellite constellation for each of the 729 configurations, we need only identify one for a single configuration from each class. If the number of classes is significantly less than the 729 original configurations then this will result in substantial computer run time savings, both in identifying all optimal constellations and in subsequent runs of performance prediction tools.

In Subsection 2.1 we count the classes, their coverage maps, and the number of classes containing a given number of satellites. In Subsection 2.2 we determine information about the structure and size of each class. In Subsection 2.3 we conclude on the utility of this approach, particularly for future constellation management.

\subsection{Number of Configuration Equivalence Classes and Coverage Maps}

This section assumes familiarity with combinatorics and group theory at the level $[4,5,6]$.

The configuration 565544 mentioned above is in an equivalence class of size 12 . The class is obtained from the action of the dihedral group $D_{6}$ on the configuration 565544 (or on any single configuration in the class). This group consists of those 12 permutations of the set $\{1,2,3,4,5,6\}$ that are equivalent to the identity permutation $\pi_{1}$ below, modulo rotation and reflection. (Formally, it is the subgroup of permutations generated by $\pi_{2}$ and $\pi_{8}$.) They are listed below, each written as a permutation and as a product of cycles.

$$
\begin{aligned}
& \text { Rotations } \\
& \pi_{1}=123456=(1)(2)(3)(4)(5)(6) \\
& \pi_{2}=234561=(123456) \\
& \pi_{3}=345612=(135)(246)
\end{aligned}
$$

$$
\begin{aligned}
& \pi_{4}=456123=(14)(25)(36) \\
& \pi_{5}=561234=(153)(264) \\
& \pi_{6}=612345=(165432) \\
& \text { Reflections } \\
& \pi_{7}=654321=(16)(25)(34) \\
& \pi_{8}=165432=(1)(4)(26)(35) \\
& \pi_{9}=216543=(12)(36)(45) \\
& \pi_{10}=321654=(2)(5)(13)(46) \\
& \pi_{11}=432165=(14)(23)(56) \\
& \pi_{12}=543216=(3)(6)(15)(24)
\end{aligned}
$$

Not every equivalence class consists of 12 configurations. As examples, the configuration 445445 is in a class of size three and 444444 is the only one in its class.

We first count the equivalence classes. This problem has sometimes been called the bracelet or free necklace problem [7] and has been studied recently [8,9]. We apply Pólya's Theorem [4 Chap. 8], which we will formally state shortly. It requires the permutation group cycle index for $D_{6}[10]$, which is

$$
Z=\frac{1}{12}\left(2 a_{6}+2 a_{3}^{2}+4 a_{2}^{3}+3 a_{1}^{2} a_{2}^{2}+1 a_{1}^{6}\right)
$$

The terms contain information about the cycles in the permutations. For example, the $4 a_{2}^{3}$ term tells us there are four permutations of $D_{6}$ that consist of the product of three 2-cycles, and the $3 a_{1}^{2} a_{2}^{2}$ term means there are three permutations that consist of two 1 -cycles and two 2-cycles. Each $a_{i}$ is a user-selected value associated with cycles of length $i$. The cycle index is a weighted average across the 12 permutations of $D_{6}$ using those values.

In the terminology of Pólya's Theorem, we regard the satellite numbers 4,5 , and 6 as colors, and view a configuration as an assignment of those colors onto the set $S=\{\mathrm{A}, \mathrm{B}, \mathrm{C}, \mathrm{D}, \mathrm{E}, \mathrm{F}\}$ of orbital planes. A special case of the theorem states that the number of orbits, or equivalence classes resulting from the dihedral group acting on the set of configurations ${ }^{1}$, is found by

\footnotetext{
1 To avoid confusion, we use "orbit" only in its astrodynamic sense and use "equivalence class" rather than "orbit" in the group theoretical sense.
} 
replacing each $a_{i}$ with the number of colors, which in our case is 3. Doing this yields $\mathbf{9 2}$ equivalence classes. The reduction from 729 configurations to 92 is nearly an order of magnitude. This is important in the use of the GPSOPT tool described in Subsection 1.2. Instead of inputting 729 configurations and searching for an optimal constellation for each, one needs only to input a representative configuration from each of the 92 classes.

This is also important in the use of performance prediction tools (Subsection 1.1), which input coverage maps. We can make the performance prediction problem tractable by restricting the number of coverage maps to those arising from the 92 optimal constellations. Each equivalence class produces six distinct optimal coverage maps, as explained here: Given an (optimal) constellation, each of the six rotations of its configuration produces a coverage map, each shifted in longitude by $60^{\circ}$. Three, however, are repeats, because of the repeating $180^{\circ}$ longitude coverage pattern. By a similar argument, the reflections produce three distinct coverage maps, for a total of six for the class. The six maps are distinct for any class, regardless of its size, because satellite arguments of latitude differ from orbital plane to plane. This holds even for a configuration such as 444444 , which is the only one in its class: it is not true that identical arguments of latitude in differing planes - in particular, uniform spacing inside of a plane - leads to optimal performance [3]. Thus there are $6 \times 92$, or 552 distinct optimal coverage maps.

Before proceeding to the next subsection we consider one more counting problem, namely, the number of classes that contain a given number of satellites. Suppose, for example, that we expect certain satellites to fail while also planning to launch additional GPS satellites until there are 32 functioning in the constellation. As we will see shortly, there are 12 equivalence classes (with a total of 90 configurations) that contain exactly 32 satellites. We could restrict our attention to those 12 classes, identify which are operationally practical, select the one with the best performance, and maneuver the satellites to attain the optimal constellation for a configuration in that class.

Let $w(r)$ be a weight assignment for each color $r$. For brevity denote

$$
x=w(4), y=w(5), z=w(6) .
$$

We replace each $a_{i}$ in the cycle index with the sum of the weights each to the $i^{\text {th }}$ power. We obtain the pattern inventory

$$
\begin{aligned}
& \frac{1}{12}\left[\begin{array}{l}
2\left(x^{6}+y^{6}+z^{6}\right)+2\left(x^{3}+y^{3}+z^{3}\right)^{2}+4\left(x^{2}+y^{2}+z^{2}\right)^{3} \\
+3(x+y+z)^{2}\left(x^{2}+y^{2}+z^{2}\right)^{2}+1(x+y+z)^{6}
\end{array}\right] \\
& =x^{6}+x^{5} y+x^{5} z+3 x^{4} y^{2}+3 x^{4} y z+3 x^{4} z^{2}+3 x^{3} y^{3} \\
& +6 x^{3} y^{2} z+6 x^{3} y z^{2}+3 x^{3} z^{3}+3 x^{2} y^{4}+6 x^{2} y^{3} z+11 x^{2} y^{2} z^{2} \\
& +6 x^{2} y z^{3}+3 x^{2} z^{4}+x y^{5}+3 x y^{4} z+6 x y^{3} z^{2}+6 x y^{2} z^{3} \\
& +3 x y z^{4}+x z^{5}+y^{6}+y^{5} z+3 y^{4} z^{2}+3 y^{3} z^{3}+3 y^{2} z^{4}+y z^{5} \\
& +z^{6} .
\end{aligned}
$$

The monomial terms, each of which has exponents summing to six, give us information about the number of equivalence classes of certain types. For example, the coefficient in the $6 x^{2} y^{3} z$ term means there are six classes for the configurations having the pattern consisting of two 4's, three 5's, and one 6. ${ }^{2}$ (For clarity, we will refer to this as a color pattern.)

Observe that, by symmetry in the unexpanded pattern inventory expression, every term with the exponents 3, 2, and 1 - that is, every term corresponding to the partition $\{3,2,1\}$ of the integer 6 has the same coefficient, namely 6 . In general, all terms corresponding to the same partition have the same coefficient.

Now we assign the weights $w(r)=s^{r}$, that is, $x=s^{4}, y$ $=s^{5}$, and $z=s^{6}$, and substitute these into the pattern inventory. We obtain the satellite (or color) inventory

\footnotetext{
2 Pólya's Theorem reads as follows: Suppose that $G$ is a permutation group acting on a set $S, Z\left(a_{1}, a_{2}, a_{3}, \ldots, a_{k}\right)$ is the cycle index for $G, C(S, R)$ is the collection of all colorings of $S$ using colors in $R$, and $w$ is a weight assignment on $R$. Then the pattern inventory of colorings in $C(S, R)$ is given by $Z\left(\sum_{r \in R} w(r), \sum_{r \in R}[w(r)]^{2}, \sum_{r \in R}[w(r)]^{3}, \ldots, \sum_{r \in R}[w(r)]^{k}\right)$.

In the special case that $w(r)=1$ for each $r \in R$ then the pattern inventory yields the number of equivalence classes.
} 
$s^{24}+s^{25}+4 s^{26}+6 s^{27}+12 s^{28}+13 s^{29}+18 s^{30}+13 s^{31}$ $+12 s^{32}+6 s^{33}+4 s^{34}+s^{35}+s^{36}$

The coefficient of $s^{k}$ is the number of classes that contain exactly $k$ satellites. For example, there are 12 classes containing exactly 32 satellites. As another example, if we wish to restrict the number of satellites to at most 31, then we are interested in just 68 of the 92 classes, corresponding to the terms with exponent at most 31 .

\subsection{Structures and Sizes of Configuration Equivalence Classes}

In this subsection we identify a representative configuration and the size for each of the 92 equivalence classes. Knowing a class size will be helpful in performance prediction tools, where the probabilities of each constellation are used. For example, if all 729 configurations are equally likely, then a class's size will determine its probability.

We begin by summarizing information from the pattern inventory in Table 1. Consider for example the row for the partition $\{4,2,0\}$. This is for classes where there are four of one color, two of another color, and none of the remaining color. There are three choices for the color that appears four times, then two choices for the color that appears twice. Thus that partition has $3 \times 2$, or 6 color patterns, each with a corresponding term in the pattern inventory with the exponents 4,2 , and 0 . Any such term, e.g. $3 x^{2} z^{4}$, has coefficient 3 , which is the number of classes having that color pattern. (Recall that every term corresponding to a given partition has the same coefficient.)

Table 1 Partitions and color patterns.

\begin{tabular}{lll}
\hline partition of 6 & \# color patterns & \# classes per color pattern \\
\hline$\{6,0,0\}$ & 3 & 1 \\
$\{5,1,0\}$ & 6 & 1 \\
$\{4,2,0\}$ & 6 & 3 \\
$\{4,1,1\}$ & 3 & 3 \\
$\{3,3,0\}$ & 3 & 3 \\
$\{3,2,1\}$ & 6 & 6 \\
$\{2,2,2\}$ & 1 & 11 \\
\hline
\end{tabular}

As shown in Table 1, there are:
- 7 partitions of the integer 6 into at most 3 parts. This is the number of ways of writing 6 as the sum of at most three positive integers - for example, $6=3+2+1$ or $6=4+2-$ where $6=n$ is the number of orbital planes and $3=m$ is the number of colors (possible number of satellites available for each plane). There is no known general closed-form expression for the number of partitions of $n$ into at most $m$ parts. (This is the same as the number of partitions of $n$ into parts where the largest is $m$, which is a well-studied type of partition [11].) For brevity we will continue to refer to these as just partitions of 6, or partitions.

- 28 color patterns. This is the sum of the second column of Table $1 .^{3}$ It is the number of terms in the pattern inventory, which is the number of ways of writing a monomial with $3=m$ variables whose exponents sum to $6=n$, which is $\left(\begin{array}{c}m+n-1 \\ n\end{array}\right)$ [4 Section 2.9].

We use partitions of 6 and color patterns throughout the subsection.

Now we investigate the sizes of the equivalence classes. We first observe that color pattern does not determine class size. For example, the configurations 555456 and 554556 have the same color pattern, but the first configuration is in a class of size 12 while the second configuration is in a class of size 6 .

Let $G_{c}$ be the set of stabilizers of the configuration $c$ : the permutations in $D_{6}$ that, when they act on $c$, leave $c$ unchanged. For example, the configuration $c=664664$ is stabilized by four permutations: $\pi_{1}$ (which is the identity permutation and stabilizes any configuration), $\pi_{4}, \pi_{7}$, and $\pi_{10}$. This is most easily seen from the configuration color position sets. For our example $c=664664$, these are $\{1,2,4,5\}$ and $\{3,6\}$, which list positions with the same color. The permutation $\pi_{4}=(14)(25)(36)$, for example, stabilizes $c$ because each of its cycles is contained in one of $c$ 's color position

\footnotetext{
${ }^{3}$ It is also coincidentally the sum of the third column. But in general the two column sums are not the same. It does not occur, for example, when we increment the number of colors to $m=4$.
} 
sets; the (numbers in the) cycle (14), for instance, are contained in $\{1,2,4,5\}$. In general, $\pi_{i}$ is a stabilizer of configuration $c$ whenever each of $\pi_{i}$ 's cycles, viewed as a set, is a subset of one of $c$ 's color position sets.

Let $\bar{c}$ be the equivalence class containing the configuration $c$, where $|\bar{c}|$ is that class's size, $\left|G_{c}\right|$ is the number of permutations in $G_{c}$, and $|G|$ is the number of permutations in the group, which in our case is 12 . The orbit-stabilizer theorem gives a formula for class size:

$$
|\bar{c}|=\frac{|G|}{\left|G_{c}\right|}
$$

For the example $c=664664$, the number of configurations in the class $\bar{c}$ is $\frac{12}{4}$, or 3 .

Rather than apply this formula to all 729 configurations, or even to all 92 classes (if one first identified a representative configuration for each class), we will apply it to configuration structures in which the variables $\mathbf{a}, \mathbf{b}$, and $\mathbf{c}$ represent different colors that can be assigned later. For example, the configuration structure aababc represents the configurations 445456 , 556564, and so on. For each partition of 6 it is straightforward to identify its configuration structure classes - that is, the classes of its configuration structures modulo rotations and reflections - along with the numbers of configuration equivalence classes and class sizes. (We now have two types of classes, configuration and [configuration] structure.) These are listed in Table 2, with a representative for each structure class. For example, consider the structure class corresponding to the row with partition $\{4,2,0\}$ and representative aabaab. (There are two other configuration structures in that structure class, abaaba and abbabb [we begin all configuration structures with a].) The number of configuration equivalence classes it represents is computed like the number of color patterns in Table 1: three choices for a's color and then two for $\mathbf{b}$ 's, so it is $3 \times 2$, or 6 . The size of each of those classes is 3 , which is computed from the number of stabilizers.

There are 22 structure classes in Table 2, a partial count of which comes from the pattern inventory term coefficients, which are shown in Table 1. For example, the partition $\{4,2,0\}$ in Table 1 has a 3 in the third column, and that partition in Table 2 has three structure classes. More generally, for all but one partition of 6 , its number of structure classes is the same as its number of configuration classes per color pattern. The exception is $\{2,2,2\}$; see Subsection 3.2 for possible future research regarding this type of partition. Also, there are 174 configuration structures, which is the sum of the last column in Table 2.

Now, in order to determine configuration class sizes or to select representative structures as inputs to GPSOPT (after assigning colors to $\mathbf{a}, \mathbf{b}$, and $\mathbf{c}$ ) to find optimal constellations, we need only reference the 22 structure classes in Table 2.

Another utility of the structure classes is in constructing constraints for the nonlinear programming component of GPSOPT to avoid repetitions of identical search spaces. Consider for example the configuration 445445. The same sequence of satellite arguments of latitude applied first to the (A-F) orbital planes and then to the (D-F,A-C) planes will yield the same overall performance, but an unconstrained nonlinear program would consider those as different constellations. Such repeat patterns are captured by the structure classes. For example, aabaab captures the repeat pattern of 445445 , as well as those of the other configurations in the six equivalence classes that it represents. We need not consider structure classes with 12 elements because they have no repeat patterns. Thus we need only identify a set of constraints for each of 13 structure classes. Subsection 3.2 suggests this as future work.

We summarize the class sizes in Table 3.

We summarize the terminology and total number associated with taxonomies we have dealt with in this section in Table 4. 
Table 2 Configuration structure classes.

\begin{tabular}{|c|c|c|c|c|c|}
\hline partition of 6 & $\begin{array}{l}\text { structure class } \\
\text { representative }\end{array}$ & $\begin{array}{l}\text { \# config. } \\
\text { classes }\end{array}$ & $\begin{array}{l}\text { color position sets (used to } \\
\text { identify stabilizers) }\end{array}$ & stabilizers & $\begin{array}{l}\text { class size: } \\
12 / \text { (\# stabilizers) }\end{array}$ \\
\hline$\{6,0,0\}$ & aаaаaa & 3 & $\{1, \ldots, 6\}$ & all $12 \pi_{i}^{\prime}$ s & 1 \\
\hline$\{5,1,0\}$ & aaaaab & 6 & $\{1, \ldots, 5\},\{6\}$ & $\pi_{1}, \pi_{12}$ & 6 \\
\hline$\{4,2,0\}$ & aaaabb & 6 & $\{1,2,3,4\},\{5,6\}$ & $\pi_{1}, \pi_{11}$ & 6 \\
\hline$"$ & aaabab & $"$ & $\{1,2,3,5\},\{4,6\}$ & $\pi_{1}, \pi_{10}$ & 6 \\
\hline$"$ & aabaab & $"$ & $\{1,2,4,5\},\{3,6\}$ & $\pi_{1}, \pi_{4}, \pi_{9}, \pi_{12}$ & 3 \\
\hline$\{4,1,1\}$ & aaaabc & 3 & $\{1,2,3,4\},\{5\},\{6\}$ & $\pi_{1}$ & 12 \\
\hline$"$ & aaabac & $"$ & $\{1,2,3,5\},\{4\},\{6\}$ & $\pi_{1}$ & 12 \\
\hline$"$ & aabaac & $"$ & $\{1,2,4,5\},\{3\},\{6\}$ & $\pi_{1}, \pi_{12}$ & 6 \\
\hline$\{3,3,0\}$ & aaabbb & 3 & $\{1,2,3\},\{4,5,6\}$ & $\pi_{1}, \pi_{10}$ & 6 \\
\hline$"$ & aababb & $"$ & $\{1,2,4\},\{3,5,6\}$ & $\pi_{1}$ & 12 \\
\hline$"$ & ababab & $"$ & $\{1,3,5\},\{2,4,6\}$ & $\pi_{1}, \pi_{3}, \pi_{5}, \pi_{8}, \pi_{10}, \pi_{12}$ & 2 \\
\hline$\{3,2,1\}$ & aaabbc & 6 & $\{1,2,3\},\{4,5\},\{6\}$ & $\pi_{1}$ & 12 \\
\hline$"$ & aaabcb & $"$ & $\{1,2,3\},\{4,6\},\{5\}$ & $\pi_{1}, \pi_{10}$ & 6 \\
\hline$"$ & aababc & $"$ & $\{1,2,4\},\{3,5\},\{6\}$ & $\pi_{1}$ & 12 \\
\hline$"$ & aabacb & $"$ & $\{1,2,4\},\{3,6\},\{5\}$ & $\pi_{1}$ & 12 \\
\hline$"$ & aabacc & $"$ & $\{1,2,4\},\{3\},\{5,6\}$ & $\pi_{1}$ & 12 \\
\hline$"$ & ababac & $"$ & $\{1,3,5\},\{2,4\},\{6\}$ & $\pi_{1}, \pi_{12}$ & 6 \\
\hline$\{2,2,2\}$ & aabcbc & 3 & $\{1,2\},\{3,5\},\{4,6\}$ & $\pi_{1}$ & 12 \\
\hline$"$ & abccba & $"$ & $\{1,6\},\{2,5\},\{3,4\}$ & $\pi_{1}, \pi_{7}$ & 6 \\
\hline$"$ & abcacb & $"$ & $\{1,4\},\{2,6\},\{3,5\}$ & $\pi_{1}, \pi_{8}$ & 6 \\
\hline$"$ & abcabc & 1 & $\{1,4\},\{2,5\},\{3,6\}$ & $\pi_{1}, \pi_{4}$ & 6 \\
\hline$"$ & aabbcc & ” & $\{1,2\},\{3,4\},\{5,6\}$ & $\pi_{1}$ & 12 \\
\hline
\end{tabular}

Table 3 Numbers of classes of each size.

\begin{tabular}{lll}
\hline class size & \# structure classes & \# config. classes \\
\hline 1 & 1 & 3 \\
2 & 1 & 3 \\
3 & 1 & 6 \\
6 & 10 & 43 \\
12 & 9 & 37 \\
Total: & 22 & 92 \\
\hline
\end{tabular}

Table 4 Taxonomies and counts.

\begin{tabular}{ll}
\hline Taxonomy & \# elements \\
\hline Configurations & 729 \\
Configuration equivalence classes / & \\
optimal constellations, modulo rotation & 92 \\
and reflection & 22 \\
Configuration structure classes & 552 \\
Optimal coverage maps & \\
\hline
\end{tabular}

\subsection{Utility of Pólya's Theorem and Our Extensions}

The reduction in constellations and coverage maps will result in significant computational savings in finding optimal constellations and in performance prediction tools. An optimization tool needs to identify only 92 optimal constellations, one for each configuration equivalence class. If we anticipate scheduling launches to attain these optimal constellations, then we only need to input 552 optimal coverage maps into performance prediction tools, which will result in ongoing computer run time savings.

We wrote a computer program that corroborated the results of this section. It lists the 92 classes and the configurations in each. Table 5 shows some entries from that output.

Because it is for just the three colors 4,5 , and 6 , the output is not difficult to assimilate. One can, for example, search for " 29 " in the "\# satellites" column to find the (thirteen) classes with exactly 29 satellites, and their sizes. With some (tedious) data manipulation on the output, we could obtain all of the information that Pólya's Theorem gave us above.

But the problem quickly becomes more complicated. If in the future we have the option of allowing 4, 5, 6, or 
Table 5 Excerpt of computer output listing equivalence classes.

\begin{tabular}{|c|c|c|c|c|c|c|c|c|}
\hline \multirow{2}{*}{$\frac{\text { class\# }}{1}$} & \multirow{2}{*}{$\begin{array}{l}\text { class size } \\
1\end{array}$} & \multirow{2}{*}{$\begin{array}{l}\text { \#satellites } \\
30\end{array}$} & \multicolumn{4}{|c|}{ configurations in class } & & \\
\hline & & & 555555 & & & & & \\
\hline 2 & 1 & 36 & 666666 & & & & & \\
\hline 3 & 1 & 24 & 444444 & & & & & \\
\hline 4 & 2 & 27 & 545454 & 454545 & & & & \\
\hline $\begin{array}{l}\cdots \\
92\end{array}$ & 12 & 33 & $\begin{array}{l}665655 \\
556566\end{array}$ & $\begin{array}{l}566565 \\
655656\end{array}$ & $\begin{array}{l}556656 \\
665565\end{array}$ & $\begin{array}{l}655665 \\
566556\end{array}$ & $\begin{array}{l}565566 \\
656655\end{array}$ & 656556 \\
\hline & & & 556566 & 655656 & 665565 & 566556 & 656655 & 565665 \\
\hline
\end{tabular}

7 satellites in any orbital plane, then we have four colors. (In fact, as of 2016, orbital plane E contains seven functioning satellites.) In that case there are $4^{6}$, or 4,096 configurations, and using Pólya's Theorem we have that there are 430 equivalence classes. If further we allow between 4 and 8 satellites in an orbital plane then we have 15,625 configurations in 1,505 classes. The problem becomes large fast, and analysis and cataloging of the classes becomes difficult. Pólya's Theorem and other combinatorial techniques may become even more useful in the future for GPS constellation management and performance optimization. See Section 3 for possible future work.

\section{Future Work}

Section 2 introduced solutions to a number of problems when there are 4,5 , or 6 satellites in each of the six orbital planes. It would be interesting, both theoretically and operationally [3], to extend these solutions to the case where there may be more (or fewer) satellites per plane, that is, where the number of "colors" $m$ is different from three, and where the number of orbital planes $n$ is different from six. Some research topics on configuration and structure classes for general $m$ and $n$ are given below.

\subsection{Properties of Configuration Equivalence Classes}

- Determine the possible configuration class sizes and explore patterns of the numbers and types of classes with each size. Consider for example the two configurations 456654 and 456456. Both are in an equivalence class of size six, but how those classes are generated are different. The first configuration yields six configurations by rotation, but no new ones by reflection. So, its class sizes relative to $D_{6}$ and relative to rotation alone, that is, relative to $C_{6}$, the cyclic group, are the same. The second configuration yields three configurations by rotation and then three more after reflection. So its class size relative to $D_{6}$ is six whereas its class size relative to $C_{6}$ is three. It would be interesting to explore such classes for general $m$ and $n$.

- For $k$ satellites, study the satellite inventory and discover patterns about the numbers of configuration classes and their sizes. Another approach would be to explore the partitioning of $k$ into exactly $n$ parts where each part is one of the possible $m$ colors, and order of the parts matters, modulo rotations and reflections.

- Explore patterns of $\omega_{m, n}$, the number of configuration classes of length $n$ and at most $m$ colors, such as recurrence formulas. Using this notation $\omega_{3,6}=92$, as we computed in Subsection 2.1. The cycle index for the dihedral group $D_{n}$ is known for any $n$ [10], from which $\omega_{m, n}$ can be computed for any $m$ and $n$ using Pólya's Theorem. Another approach would be to define $\sigma_{m, n}$ as the number of classes of length $n$ and exactly $m$ colors, explore its properties, then use the equation $\omega_{m, n}=\sum_{i=1}^{\min (m, n)}\left(\begin{array}{c}m \\ i\end{array}\right) \sigma_{i, n}$. It is straightforward that $\sigma_{m, m}=(m-1) ! / 2$ for $m \geq 3$, but other values of $\sigma_{m, n}$ are more difficult to find. This or some other combinatorial approach may result in characterizing the values of $\sigma_{m, n}$ and $\omega_{m, n}$. 


\subsection{Properties of Structure Classes}

- Discover a technique that identifies and counts the structure classes. It was straightforward to identify the 22 structure classes for the case $m=3$ and $n=6$ shown in Table 2, but it would be more difficult for larger $m$ and $n$. The study of the problem just below will help in counting the structure classes.

- Study those partitions of integers where the number of structure classes differs from the number of configuration classes per color pattern; see the comment in Subsection 2.2 in the explanation of Table 2. This occurs in the partition $\{2,2,2\}$ in which colors appear the same number of times and its structure classes represent fewer configuration classes than there are assignments of colors to the variables. For example, the structure class containing abcabc represents just one configuration class, even though there are six different assignments of the colors 4, 5, 6 to the variables a, b, c. This is also why the numbers of configuration classes for that partition are different: some of its structure classes represent three configuration classes and others represent just one. For $m=3$ and $n=6$ the partition $\{2,2,2\}$ is the only one for which this complication occurs, but for general $m$ and $n$ there are probably different numbers of such partitions of $n$ into at most $m$ parts.

- Construct a set of nonlinear programming constraints for each of the 13 structure classes with repeat patterns that were introduced in Subsection 2.2. These constraints can then be incorporated into GPSOPT.

\section{Conclusion}

In this study we utilized the symmetry of the equally-spaced orbital planes and Pólya's Theorem to reduce the number of configurations on which to optimize from 729 down to 92 equivalence classes. Each class produces six optimal coverage maps, for a total of 552, which can be input into performance prediction tools. We counted classes containing a given number of satellites. We identified 22 structure classes and applied combinatorial and group theory techniques such as the orbit-stabilizer theorem to determine the size and number of configuration classes for each.

Future work may include studies of properties of the equivalence and structure classes, particularly if we allow more than six satellites per plane (or fewer than four), which could be of both operational and theoretical interest.

\section{Acknowledgments}

Some of the work for this paper was done while the first named author was on sabbatical at and supported by Headquarters, Air Force Space Command, Advanced Space Analysis Division HQ AFSPC/A9Y. We thank Mike Ferrara, Department of Mathematical and Statistical Sciences at University of Colorado Denver, for suggesting the use of the variable $s$ in the satellite inventory in Subsection 2.1. We also thank Beth Schaubroeck, Department of Mathematical Sciences at the U. S. Air Force Academy, for reviewing the paper.

\section{References}

[1] Langley, R. B., "Dilution of Precision," GPS World, May 1999, pp. 52-59, URL:

http://www.nrem.iastate.edu/class/assets/nrem446_546/w eek3/Dilution_of_Precision.pdf [cited 29 July 2014].

[2] Kaplan, E. D. and C. J. Hegarty (Eds.), Understanding GPS: Principles and Applications, $2^{\text {nd }}$ ed., Artech House, Boston, 2005, Section 7.3.

[3] Peterson, D. C., S. P. Rinaldi, and M. F. Storz, "Global Positioning System Performance Optimization using a Normalized Function on Configuration Classes," Journal of Guidance, Control, and Dynamics, to appear.

[4] Roberts, F. S. and B. Tesman, Applied Combinatorics, $2^{\text {nd }}$ ed., CRC Press, Boca Raton, Florida, 2009, Chaps. $1-2,8$.

[5] Gallian, J. A., Contemporary Abstract Algebra, $8^{\text {th }}$ ed., Brooks/Cole Cengage Learning, Boston, 2012, Chaps. 0-2, 5, 7, 29.

[6] Hungerford, T. W., Algebra, Springer, Berlin, 1974, Section II.4.

[7] Weisstein, E. W., "Necklace," from MathWorld-A Wolfram Web Resource, URL:

http://mathworld.wolfram.com/Necklace.html [cited 15 December 2014]. 
[8] Badar, M. and A. Iqbal, "Pólya's Enumeration Theorem," Masters thesis, School of Computer Science, Physics, and Mathematics, Linnaeus University, Sweden, June 2010, URL:

http://lnu.diva-portal.org/smash/record.jsf?pid=diva2\%3 A324594\&dswid=-2813 [cited 15 December 2014].

[9] Fel, L. G., "On the Pólya Enumeration Theorem,"
Intelligent Information Management, Vol. 1, 2009, pp. 172-173.

[10] Harary, F., Graph Theory, Westview Press, Boulder, Colorado, 1994, pp. 181 and 184.

[11] Andrews, G. E., The Theory of Partitions, Cambridge University Press, Cambridge, Massachusetts, 1998, Chaps. 1, 2, 13 . 